54. Jahresversammlung der Schweizerischen Gesellschaft für Dermatologie und Venereologie 30. September und 1. Oktober 1972 in Genf

Dermatologica, 1973;147:45

\title{
Neues zur Therapie der Gonorrhö
}

\begin{tabular}{|l|l|}
\hline F. & Gilliet \\
\hline H. & Storck \\
\hline
\end{tabular}

Dermatologische Universitätsklinik (Direktion: Prof. H. Storck), Zürich

Sonderuckbestelllungen an: Prof. Dr. med. H. Strock, Dermatilogische Universitätsklinik, Gloriastrasse 31, CH-8006 Zürich (Schweiz)

Zusammenfassung

Es sind die mit verschiedenen Antibiotika bei der Therapie der akuten Gonorrhö in den letzten Jahren veröffentlichten Resultate zusammengestellt. Für Penicillin konnte aus der statistischen Betrachtung eine genaue Dosis-Zeit-Relation aufgestellt werden. So ist zusätz-lich zum schnell ansteigenden hohen Initialspiegel im Blut ein bis drei Tage dauernder Depoteffekt für beste Resultate wünschenswert. Diese Forderungen werden durch die gleichzeitige perorale Gabe von Probenecid, welches die Penicillinausscheidung durch die Nieren hemmt, am einfachsten erfüllt. Neben Penicillin und Ampicillin sind Thiamphenicol per os und Spectinomycin intramuskulär für die Einzeittherapie als gleichwertige Alterna-tiven zu betrachten. Ferner kommen auch Kanamycin, Erythromycin, Gentamycin, Rifampi-cin und Cephaloridin, in Einzelfällen auch Tetrazykline in Frage, mit immer noch guten Resultaten. Genaue Dosierung sowie Fragen der Kontrollen über den Heilungsverlauf werden besprochen. Sicherheitshalber empfehlen wir noch immer die serologische Lues-kontrolle bei alien Patienten zwei Monate nach der Therapie. 1 Erscheint in extenso in der Schweizerischen medizinischen Wochenschrift. 